\title{
LIMITATIONS OF DESIGN SPACE-BASED INDICATORS FOR EARLY ROBUSTNESS ASSESSMENT
}

\author{
Juul-Nyholm, Herle Bagh (1); \\ Sigurdarson, Nökkvi S. (1,2); \\ Ebro, Martin (2); \\ Eifler, Tobias (1) \\ 1: Technical University of Denmark; \\ 2: Novo Nordisk A/S
}

\begin{abstract}
This paper seeks to address the gap between qualitative Robust Design principles and parameter optimization. The former often fails to consider the challenging amount of details in embodiment and configuration design, while the latter is the widely accepted main thrust in traditional Robust Design. The gap is addressed by exploring the value of five quantitative robustness indicators for Design Space Exploration based on variables, objectives and constraints: The set level indicators, Design Space Size and Pareto Set Dispersion, and the point level indicators, Neighbourhood Performance, Failure Rate and Distance to Failure. As a background for the discussion of the limitations of these indicators an industrial case is presented. The case is an incremental encoder and includes two configurations for comparison, five objectives, eight variables, and a range of constraints. The design spaces are sampled and they show conflicting objectives, dispersed spaces and variables dependencies. Based on this it is suggested that set level indicators are more suitable than point level indicators of early robustness evaluation, but the available indicators are limited in their considerations of design space discontinuity and conflicts.
\end{abstract}

Keywords: Robust design, Computational design methods, Evaluation, Design Space Exploration, Configuration Design

\section{Contact:}

Juul-Nyholm, Herle Bagh

Danmarks Tekniske Universitet / Technical University of Denmark

Denmark

hbaju@mek.dtu.dk

Cite this article: Juul-Nyholm, H. B., Sigurdarson, N. S., Ebro, M., Eifler, T. (2021) 'Limitations of Design Space-Based Indicators for Early Robustness Assessment', in Proceedings of the International Conference on Engineering Design (ICED21), Gothenburg, Sweden, 16-20 August 2021. DOI:10.1017/pds.2021.459 


\section{INTRODUCTION}

Well-accepted in academia and practice, Robust Design (RD) provides an approach for ensuring the "insensitivity of products and processes against different sources of variation" without eliminating the sources of variation themselves (Taguchi, 2005). Seemingly offering a comprehensive development procedure, based on the three phases of the seminal quality engineering framework (1) System Design, (2) Parameter Design, and (3) Tolerance Design, RD consequently aims at developing products that show a consistently high quality and performance despite noise factors such as production variation in form of tolerances, unintended or variable load scenarios, ambient conditions of use, etc.

However, most authors agree that Taguchi's work on phase (2), i.e. the optimization of parameter settings by means of suitable experimentation strategies and the corresponding statistical analyses, is the main thrust in a traditional RD approach (e.g. Jugulum \& Frey, 2007; Hasenkamp et al., 2009). Unfortunately, this implies a relatively narrow focus on one single, albeit important, design task in embodiment, which is the efficient optimization of parameter settings for a previously defined product configuration. As a consequence, traditional RD almost exclusively focuses on the time-intensive and often computational costly optimisation of parameter settings of a largely matured product solution, and hence completely ignores the possibility of improvements by design. This question is instead left to qualitative, early stage RD principles (Blanding, 1999; Suh, 2001), which oftentimes fail to address the challenges that come with the increasing level of details in embodiment and configuration design.

This paper seeks to address this decisive gap between early stage design principles and late stage optimisation approaches by exploring the value of quantitative Design Space-based indicators for an early robustness assessment in configuration design. The underlying reasoning, that these indicators could be applied to evaluate and compare configurations of a chosen concept, has a twofold basis. On the one hand, corresponding approaches allow for an integrated consideration of a product's functionality (objectives) and its structural characteristics (imposed constraints). Examples for the latter are geometric tolerance chains, which are largely relevant for the overall robustness, but often only considered towards the end of the development process. On the other hand, they also provide the possibility to extend conventional RD-thinking towards a rigorous considerations of resulting trade-offs, which have previously been identified as a largely relevant driver of product robustness (Göhler \& Howard, 2015; Göhler, Frey \& Howard, 2016b; Sigurdarson et al., 2019). As a consequence, the overall aim is to enable the engineering designer to avoid unnecessary iterations and to proceed to the embodiment task with confidence in the chosen configuration. In other words to improve robustness by design!

\section{BACKGROUND AND METHODOLOGY}

Design Space Exploration (DSE) is the iteration and exploration of the design or variable space (Fig. 1a) of feasible design points and the corresponding objective space (Fig. 1b) defined by objective functions describing the performance of a design configuration. The variable space is defined by the variable ranges and each design point is a set of variables and objective measures subject to design specific constraints. The design points fulfilling the constraints are feasible. In the objective space, the design point performance is evaluated based on optimality, i.e. either maximum or minimum. If two objectives cannot be optimal at the same time, as can be seen in Fig. 1b, the two objectives are conflicting. The conflict is represented by the Pareto frontier, or its approximation, on which none of the objectives for each point can be improved without deteriorating another.

While the variable space represents possible parameter settings, including potential variation, the correlation between variables and objectives represents sensitivity and the objective space represents the design output possibilities and the trade-offs inherent in the design. The latter, also referred to as contradictions, is of particular interest in the context of this paper. Based on the predictive value of trade-offs as robustness indicator for early product solutions (Göhler \& Howard, 2015; Göhler, Frey \& Howard, 2016b), DSE bears the potential to be used as early stage, quantitative RD assessment of configurations, and in this way to provide a valuable complement to the detailed sensitivity studies of traditional RD approaches.

The review of existing robustness indicators in the field of DSE started by identifying the relevant body of literature. Based on an initial literature review, which yielded a collection of 74 publications on indicators, nine publications containing the search terms robust and Pareto were identified. Subsequently, 


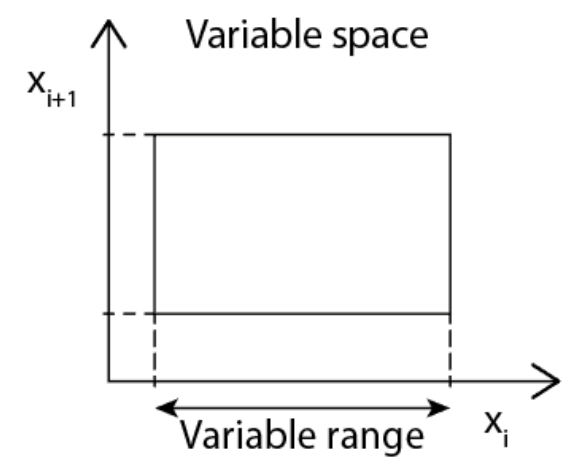

(a) Variable space and variable range for the variables $x_{i}$ and $x_{i+1}$.

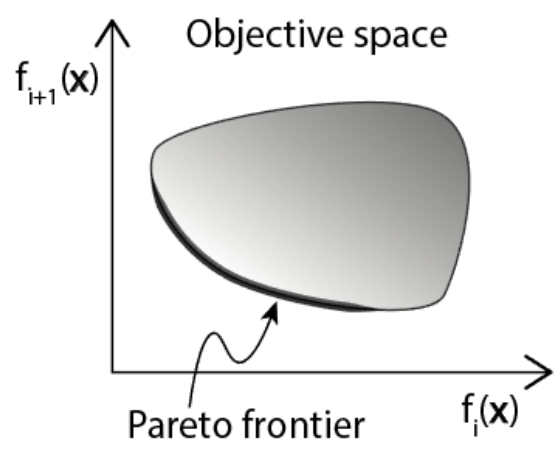

(b) Objective space and Pareto frontier for minimization of the objectives $f_{i}$ and $f_{i+1}$.

Figure 1. Visual definitions of the $2 D$ variable and objective spaces.

the indicators were grouped according to their information content, i.e. the necessary information for their calculation and the output provided, resulting in the classification presented in Sec. 3 below.

In order to discuss the challenges of the listed indicators, an exemplary case study from industry is presented. This anonymized case involves the design of an angular encoder. The case study is set up as a sampled design space exploration model to compare two encoder configurations. It was chosen as it combines multiple engineering disciplines and the design is subject to a range of demanding constraints. Furthermore, both constraints and objectives require evaluation of analytical expressions as well as simulations due to the incremental nature of the encoder scale. The chosen variables are also both continuous and discrete, highlighting the strengths of a design space exploration approach. All of this makes the case suitable for a discussion of robustness indicator limitations. Variables, objectives and constraints were identified through semi-structured interviews with design engineers in the development project, and the results are visualized by their design and objective spaces for easy comparison.

Based on the presented case study, the limitations of the identified indicators are discussed. The discussion is based on the extend to which the indicators address the challenges of the case, the required information and the computation effort required.

\section{REVIEW OF ROBUSTNESS INDICATORS FOR DESIGN SPACE EXPLORATION}

In Tab. 1 five indicator types are listed and illustrated. In the following, the short descriptions of Tab. 1 is elaborated and the indicators' implications on robustness is introduced.

The size of the design space represents the ranges of feasible design variables as a function of the constraints. Opposed to the shortest distance to a constraint, the design space size indicates the room for maneuver in choosing design points with the achievement of the maximum allowable tolerance at the center of the design space. The design space size can be evaluated as the full hypervolume of the design space as indicated in the illustration or as a box of independent variable ranges.

The size of the Pareto set on the other hand, indicates how contradictory the objective functions are in the feasible design space. It is calculated as the hypervolume between the Pareto frontiers in the objective space represented by the black edged volume in the illustration. In design space exploration the Pareto set can only an approximated as this method does not allow an evaluation of whether the set fulfils mathematical optimality criteria.

Neighbourhood performance provides an extended view on the sensitivity of objective performance to perturbations in a single variable by evaluating the performance of a specified variable neighbourhood of a design point. The neighbourhood performance can be evaluated as the average performance of the neighbourhood, which would allow for inclusion of effects of non-linear objective functions, or the worst case performance of the neighbourhood.

Failure rate is a measure of how many parts will violate constraints based on the variable distribution. The failed outcomes are represented by the dark shaded part of the box in the illustration. Rather than focusing on the worst case scenario as some neighbourhood performance indicators, it relates the nominal design point statistically to large scale production outcomes. 
The shortest distance from a point to failure indicates the maximum size of a design point tolerance, which has implications for the required production control. It can be measured either on specific variable axes or as a Euclidean distance as suggested by the arrows in the illustration.

Table 1. List of identified robustness indicators for design space exploration. References in right column: [1] (Göhler, Eifler \& Howard, 2016a), [2] (Beyer et al., 2007), [3] (Harbrecht et al., 2019) [4] (Rötzer et al., 2020), [5] (Riquelme et al., 2015), [6] (Barrico et al., 2006),

[7] (Yannou et al., 2007), [8] (Frank et al., 2018), [9] (Deb et al., 2006)

\begin{tabular}{ll}
\hline$\# \quad$ Indicator \& Short Description \\
\hline Indicators on Set Level \\
I Design Space Size \\
The n dimensional volume of the design space, \\
either in terms of range-based, independent \\
boundaries or the total space size. It indicates the \\
room for maneuver in choosing a design point and \\
hence the global robustness of the design. It requires \\
suggested variable ranges, objective functions and \\
constraints.
\end{tabular}

\section{Pareto Set Dispersion}

The $\mathrm{n}$ dimensional volume of the objective space between the Pareto frontiers between all objective pairs. It indicates how conflicting the objective functions are for the current design configuration. It requires suggested variable ranges, objective functions and constraints.

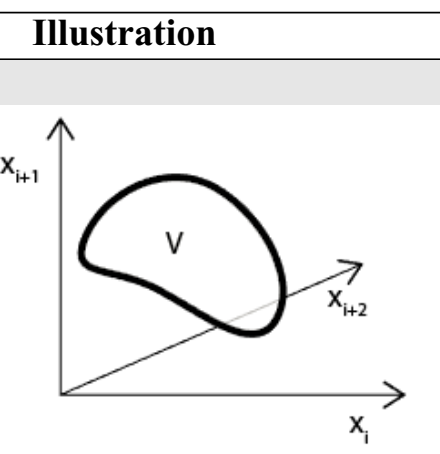

[1][2]

$[3][4]$ Ref.

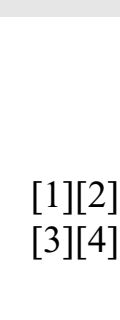

\section{Indicators on Point Level}

\section{Neighbourhood Performance}

The objective performance of the chosen neighbourhood of the design point based on either average, variance or worst case. This indicates the sensitivity of the chosen set as it describes the correlation between the design and the objective space. It requires a design point, desirable tolerances as well as the variable set and

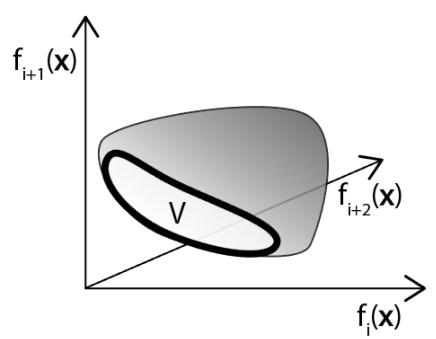
the objective functions.

\section{Failure Rate}

The rate of outcomes failing due to violation of constraints for the chosen variable space and variation distribution type. This indicates the scrap rate of a mass produced product at the chosen, nominal design point. It requires the design point, constraints, objective functions and distribution characteristics.

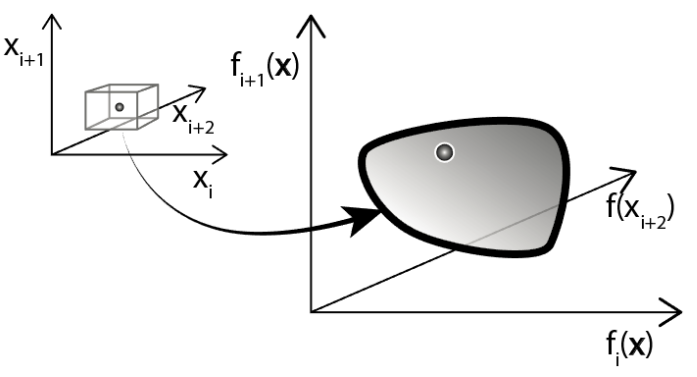

\section{Distance to Failure}

The distance from the chosen design point to the closest constraint, either in terms of variable units or as a Euclidean distance. This indicates the quality margin of the chosen design point and hence the local robustness of the design. It requires a design point, objective functions and relevant constraints.
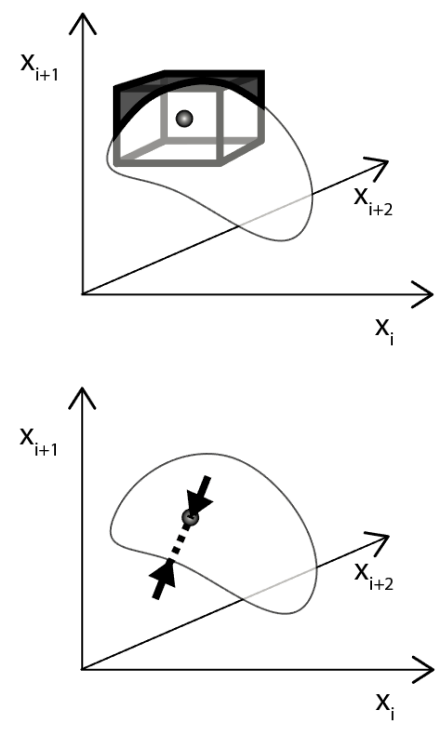


\section{ENCODER CONFIGURATION CASE}

In the following, a configuration case study for an encoder will be introduced and used to assess the applicability of the indicators identified in Sec. 3.

The case study focuses on the physical configuration of the reader and scale of an incremental, conductive encoder that is retrofitted into an existing mechanical device to register and transmit angular displacements. The reader is produced from sheet metal through cutting, bending and punching and the scale pads are separate conductive pads on the surface of a print board. The displacements correspond to critical events and the aim of the retrofit is to improve the functionality of the mechanical device by offering a digital logging of these events. Furthermore, the mechanical device is mass produced and a robust design is of paramount importance.

The fact that the encoder is an add-on leads to a series of predetermined size and functionality constraints. The encoder cannot be serviced, calibrated or replaced, and the cost has to be kept at an absolute minimum, and needs to be produced in an enormous volume (above $10^{7}$ units/year). On top of this, the displacement that needs to be measured, are incremental and have a predefined maximum range, which means that the outcomes are a known discrete set with its own unit.

Exemplified in Fig. 2, the encoder is of the conductive and incremental type as the registration of displacement is achieved as a current conducting reader is sliding over a scale consisting of separate conductive pads representing logically interpretable code digits. Hence, the rotational, mechanical input is converted to an electrical code, which is interpreted by the software as an angular displacement corresponding to a number of units belonging to the outcome set.

The configuration should maintain the original mechanical function of the device to the largest extent possible while ensuring a adequate measuring accuracy including electrical signal quality and logical code interpretability. These demands cannot be achieved without compromises. Yet, the required degree of compromise depends on the choice of the encoder configuration.

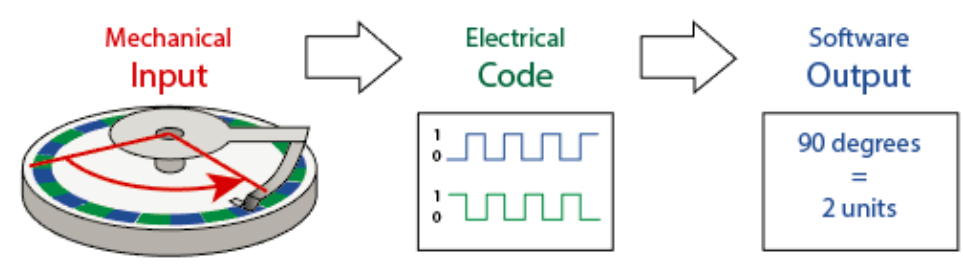

Figure 2. Encoder working principle.

The aim of this case study is to compare the robustness potential of two encoder configurations. These are shown in Fig. 3a-3b. Configuration 1 has one scale track, while Configuration 2 has two scale tracks. The white pads represent the ground connection which is essential for the conduction of current. The colored pads represent different code pads, like the green and blue pads in Fig. 2.

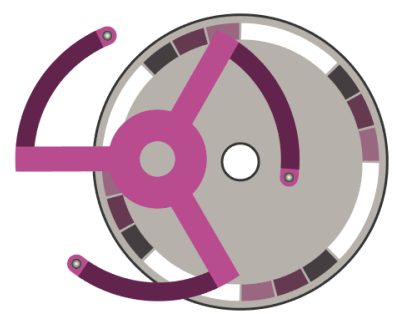

(a) Configuration 1 with three arms on one track and different code pads with grounding in between.

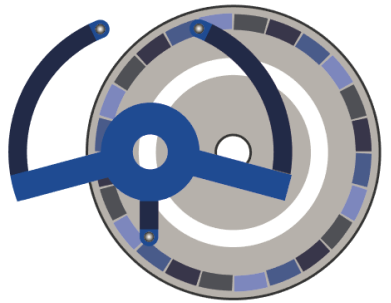

(b) Configuration 2 with two arms on the four code track and a separate track and arm for grounding.

Figure 3. Encoder configurations.

\section{DESIGN SPACE EXPLORATION FOR ENCODER CONFIGURATION}

In this section the model variables, objectives and constraints are described. They are based on a number of assumptions and therefore the results offer a means of comparison of the two configurations in 
Fig. 3a-3b. The encoder configurations were explored in MATLAB by looping through the objective functions with two identical sets of random design point samples. Subsequently, the feasible samples were identified as well as the local Pareto optimal samples for each configuration.

\subsection{Variables}

The embodiment of the configurations were varied based on the eight design variables illustrated in Fig. 4. The dimensional variables subject to variation are sampled randomly and uniformly within a set of chosen limits. They represent both noise factors and control parameters as there is no need to distinguish between them when analyzing for dependencies (Göhler, 2017). All variables are continuous except the number of scale pads, $m$, which divides the configurations into subconfigurations. Design parameters like material properties, production capabilities etc. are included in the model as well.

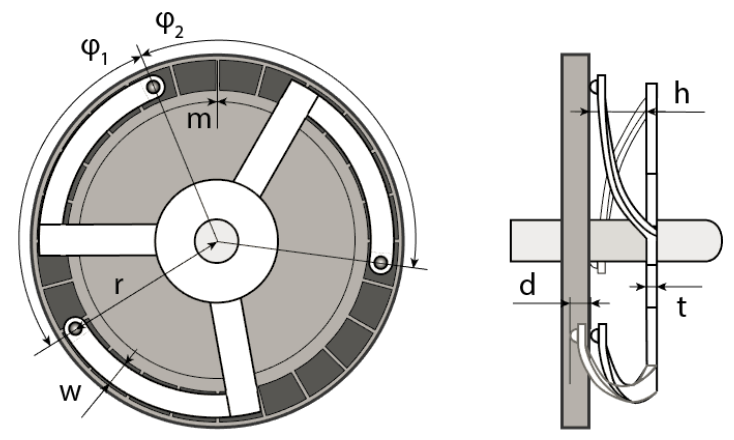

Figure 4. Configuration model variables.

\subsection{Objectives}

The objectives of the encoder design are to impact the original function of the mechanical device as little as possible, to ensure electronic registration of angular displacement as well as robustness against variation in the position of reader and scale.

Four of the five design objectives are analytical expressions subject to a multitude of assumptions but also computationally inexpensive. For modelling of encoder outputs however, simple simulations were developed. This was possible because the design space is explored by sampling.

The height of the encoder should be minimized to have as small an impact on the existing components of the mechanical device as possible. Due to the focus on the reader design, the total encoder height only varies with the thickness of the wiper plate, $t$, and the distance from the reader plate to the PCB surface, $h$ (Eq. 1).

Minimization of the frictional torque of the reader due to the scale contact, seeks to ensure as small an effect on the displacement to be measured due to the addition of the encoder (Eq. 2).

Sensitivity to swash (tilting between reader and scale planes) is undesirable because the signal strength is a function of the contact force. Assuming a constant tilting angle, $\phi$, to obtain a relative objective, the difference between the minimum and maximum contact force is a function of the contact radius, $r$. The contact forces are calculated assuming a constant tilting angle and assuming that the reader arms are straight and slender with rectangular cross sections. It is desired that the minimum and maximum contact forces are equal, which is why the objective function ratio is to be maximized (Eq. 3).

Minimization of constriction resistance is representing the function of the electrical code circuits connected to the scale pads. The lower the resistance the lower the required power. In the interface between the reader arm and the scale, the contact resistance is a sum of the constriction and the film resistance. The constriction resistance occurs because the current is constricted to travel through a reduced area across the rough interface, while film resistance is caused by a thin layer of dirt and oxides on the surfaces, which has a higher resistivity than the conductive bulk material. The film and bulk resistances of the conductive materials are not included in the model as it hard to quantify and will be apply to both configurations. The constriction resistance is a function of this radius and the resistivities of the reader and scale pad surfaces, which are the same (Eq. 4).

The maximization of repeatability in terms of the number of pads per unit depending on the starting point is important in order to avoid ambiguities in the code interpretation. The challenge of repeatability is 
pronounced in the case of this particular encoder, because the encoder can neither be calibrated during production nor use. The main contributor to the repeatability challenge, apart from dynamic effects, is the random starting position of the wiper arms on the scale pattern. The repeatability objective is evaluated by simulating rotations of 1-30 units for a range of starting positions and counting the number of pads passed. The most frequent pad count per dose, i.e. what the controller would be programmed to interpret as one specific dose, is used as a reference when calculating the repeatability for each dose. Because the objective is to be maximized, the lowest calculated repeatability among the doses is chosen as the objective measure for one particular sample. The number of starting positions where chosen based on a convergence study (Eq. 5).

$$
\begin{aligned}
& \min t+h \\
& \min T_{\mu} \\
& \max \frac{F_{\min }}{F_{\max }} \\
& \min \frac{\rho}{2 r_{a}} \\
& \max \text { Rep. }
\end{aligned}
$$

\subsection{Constraints}

The encoder configurations are subject to the following constraints. The configuration embodiment should:

1. ensure constant grounding.

2. ensure a continuous code signal on a full rotation.

3. ensure any unit interpretation regardless of the starting point on the scale.

4. not exceed the maximum yield stress of the reader arm material.

5. not exceed the minimum requirement for the contact resistance.

6. not exceed the allowable diameter of the retrofit in the mechanical device.

7. be manufacturable in terms of reader and pad dimensions.

8. ensure rotation despite friction loss due to the reader arm contact.

\subsection{Results}

The DSE model was run with $2 \cdot 10^{6}$ design points distributed uniformly across a range that complies with the size requirements and was identified through interviews. The number of feasible solutions for Configuration 1 and 2 were 562 and 176 respectively. The results are presented in Fig. 5 as selected variable and objective spaces, respectively, including all eight variables and all five objectives. The visual interpretation of these will be discussed in Sec. 6 .

\section{DIscussion}

\subsection{Case Results}

Four selected variable spaces for Configuration 1 and 2 can be seen in the upper part of Fig. 5. On the top left a dependency between the thickness, $t$, and the deflection, $d$, can be sensed for both configurations, though stronger for Configuration 1. The global optima are quite evenly distributed in $d$, but for $t$ the optimal tendency is on the low end of the range. On the top right of the variable space plots, the number of scale pads per revolution can be seen in relation to the reader height. The discrete steps in $m$ each represent subconfigurations of Configurations 1 and 2 and for Configuration 1 only four out the seven are feasible due to differences in division of three and four digit codes. Not only does Configuration 2 have one third of the number of feasible solutions compared to Configuration 1, its solution space is also more dispersed, especially in $t$ and $h$, and might be discontinuous. The discontinuity can however not be assessed due to the combination of the sampling size and the inclusion of the discrete variable, $m$. On the bottom left of the variable space plots, both feasible and globally optimal design points are dispersed across the sampled design ranges for both configurations in $w$ and $r$. This might imply discontinuity, but also shows little dependency to optimality as the global Pareto points are dispersed 

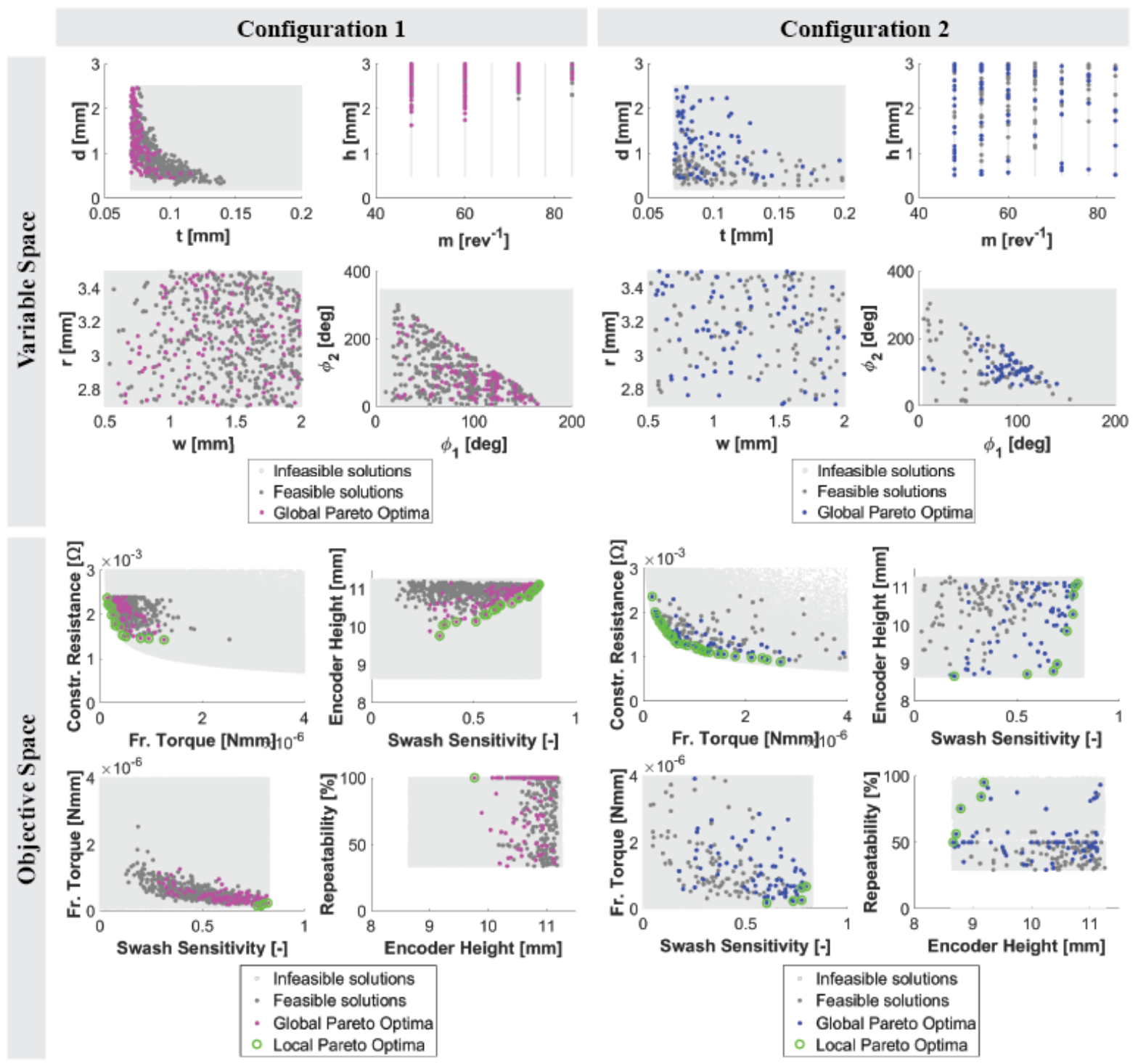

Figure 5. Variable and objective pairs for Configuration 1 and 2 including infeasible sets, feasible sets, global Pareto sets and local Pareto sets.

as well. On the bottom right of the variable space plots, the expected dependency induced by one of the manufacturability constraints (7.) creates a triangle between $\phi_{1}$ and $\phi_{2}$. For both configurations, clusters of feasible points can be seen, especially the three bands between 100 and 150 degrees for Configuration 1 . This might be related to discontinuity due to either subconfigurations or constraints related to the discrete encoder scale.

In the lower part of Fig. 5 four selected objective spaces for Configuration 1 and 2 can be seen. On the top left, the set of grey, infeasible points shows that the objective space is shaped by the relationship between the Frictional Torque and the Constriction Resistance. On the top right and bottom left of the objective space plots the infeasible spaces are square, indicating that the concept and configuration does not predispose a conflict between the objectives. On the top right, however, the Pareto front is placed very differently in the space for the two configurations due to a difference in the influence of constraints. In both top right and bottom left, the feasible Swash Sensitivity objective is dispersed along the range for both Configurations, while this is only the case for the Encoder Height and Frictional Torque for Configuration 2. This might indicate design space discontinuity or a high sensitivity to variables, but this cannot be derived from the plots. In the bottom right of the objective space plots the Repeatability objective is clearly different for the two configurations. The majority of the feasible design points for Configuration 2 are below 50\% repeatability and the Pareto set indicates a conflict, but with the scattered feasible set the objective space size is hard to assess visually. 
As mentioned in Sec. 2, this case is a challenging design task due to the number of constraints and objectives, and as shown in the variable space plots of Fig. 5 the constraints and objectives lead to both conflicts and limited design spaces for both configurations.

\subsection{Limitations of Robustness Indicators}

The five indicators listed in Tab. 1 each have their strengths and weaknesses and are applicable at different design stages due to differences in required in- and outputs, as can be seen in Tab. 2. Indicator I and II requires variable ranges as input, while Indicators III-V requires a design point. While III and IV requires tolerances as inputs, I and V provides information about feasible tolerances. On the output side, I and II consider global configuration characteristics, while III-V consider local information directly related to tolerances as well as sensitivity and scrap. Depending on the defined neighbourhood, III might however also reflect global characteristics, for example in cases of monotonicity. Based on this, the set level (I,II) indicators are applicable for coarse exploration of variable feasibility and conflicts, while the point level (III-V) indicators more often support the evaluation of a chosen design point by offering a detailed variation performance for more mature designs.

Table 2. Summary of limitations of robustness indicators for design space exploration.

\begin{tabular}{lccccc}
\hline \multicolumn{1}{c}{ Indicator } & $\begin{array}{c}\text { I } \\
\text { Design } \\
\text { Space } \\
\text { In-/Output }\end{array}$ & $\begin{array}{c}\text { II } \\
\text { Pareto } \\
\text { Set } \\
\text { Dispersion }\end{array}$ & $\begin{array}{c}\text { III } \\
\text { Neighbourhood } \\
\text { Performance }\end{array}$ & $\begin{array}{c}\text { IV } \\
\text { Failure } \\
\text { Rate }\end{array}$ & $\begin{array}{c}\text { V } \\
\text { Distance } \\
\text { to } \\
\text { Failure }\end{array}$ \\
\hline Variable ranges & Input & Input & Input & Input & Input \\
Objective Functions & Input & Input & Input & Input & Input \\
Constraints & Input & Input & Input & Input & Input \\
$\begin{array}{l}\text { Design point } \\
\text { Tolerances }\end{array}$ & (Output) & & Input & Input & Output \\
Variation Distributions & & & Input & \\
$\begin{array}{l}\text { Sensitivity } \\
\text { Scrap Rate }\end{array}$ & & & Output & Output & \\
$\begin{array}{l}\text { Optimality } \\
\text { Conflict }\end{array}$ & & Output & Output & & \\
\hline
\end{tabular}

The case and DSE model presented in Sec. 4-5 show discontinuity due to the constraints and the discrete variable (number of scale pads, $m$ ), which will result in issues both for set and point level indicators. The set level indicators would need to divide the spaces into continuous ones and the point level indicators would be unsuitable for the identification of alternative spaces. Furthermore, the case study shows two different kinds of objective conflicts; objective function and constraint induced. None of the indicators take this difference into account even though it provides important information about how to manipulate the design space and hence the obtainable robustness, e.g. through changing the configuration. The objective conflicts are also interesting on the topic of dispersion, i.e. the length of the Pareto frontiers, as it might indicate the sensitivity of the design depending on the dispersion of the corresponding variable space. This is not covered in the set level indicators either.

The presented robustness indicators differ from Taguchi's work on parameter design as they focus on robustness of multiple configurations in the early embodiment stage based on trade-offs and design flexibility as opposed to optimization of one configuration that has already been matured. This is supported by the correlation between trade-offs and robustness identified by Göhler, Frey \& Howard (2016b) and its relevance is further highlighted by the multidimensional and discontinuous design spaces of the exemplary encoder configurations. Exploration of design spaces and conflicts through the reviewed indicators could increase the predictability of designs for further detailing and optimization.

The presented review and discussion of robustness indicators for DSE was based on theory and the challenges highlighted by the industrial case. It would however be interesting to implement and evaluate the indicators quantitatively and maybe even apply them to a set of diverse cases to assess their applicability based on degree of dispersion, discontinuity or conflict as well as design space size and objective types. 


\section{SUMMARY}

The gap between early and late stage RD has been addressed through a review and classification of available indicators for DSE. Five indicators were identified: Two set level indicators, Design Space Size and Pareto Space Dispersion, and three point level indicators, Neighbourhood Performance, Failure Rate and Distance to Failure. Based on an industrial case with two encoder configurations highlighting conflicting objectives and demanding constraints as well as a table showing indicator in- and outputs, a discussion of the limitations of the five indicators were presented. For early evaluation of configuration robustness, set level indicators are more suitable than point level indicators, but the available indicators are limited in their considerations of design space discontinuity, conflicting objectives due to constraints or objective relationships as well as the robustness implications of the shape of Pareto frontiers.

\section{ACKNOWLEDGMENTS}

The authors would like to thank Novo Nordisk A/S for financial support to the DTU-NN Robust Design program.

\section{REFERENCES}

Barrico, C. \& Antunes, C. H. (2006) Robustness Analysis in Multi-Objective Optimization Using a Degree of Robustness Concept. 2006 IEEE Congress on Evolutionary Computation, 1887-1892.

Beyer, H. G. \& Sendhoff, B. (2007). Robust optimization - a comprehensive survey. Computer methods in applied mechanics and engineering, 196(33-34), 3190-3218.

Blanding, D. L. (1999). Exact constraint: machine design using kinematic principles. American Society of Mechanical Engineers.

Deb, K. \& Gupta, H. (2006). Introducing robustness in multi-objective optimization. Evolutionary computation, 14(4), 463-494.

Frank, C. P., Marlier, R. A., Pinon-Fischer, O. J., \& Mavris, D. N. (2018). Evolutionary multi-objective multi-architecture design space exploration methodology. Optimization and Engineering, 19(2), 359-381.

Göhler, S. M. \& Howard, T. J. (2015). The contradiction index (ci): a new metric combining system complexity and robustness for early design stages. International Design Engineering Technical Conferences and Computers and Information in Engineering Conference (Vol. 57175, p. V007T06A023). American Society of Mechanical Engineers.

Göhler, S. M., Eifler, T., \& Howard, T. J. (2016) Robustness metrics: Consolidating the multiple approaches to quantify robustness, Journal of Mechanical Design, 138(11).

Göhler, S. M., Frey, D. D., \& Howard, T. J. (2016). A model-based approach to associate complexity and robustness in engineering systems. Research in Engineering Design, 28(2), 223-234.

Harbrecht, H., Tröndle, D., \& Zimmermann, M. (2019). A sampling-based optimization algorithm for solution spaces with pair-wise-coupled design variables. Structural and Multidisciplinary Optimization, 60(2), 501-512.

Hasenkamp, T., Arvidsson, M., \& Gremyr, I. (2009) A review of practices for robust design methodology, Journal of Engineering Design, 20(6), 645-657.

Jugulum, R. \& Frey, D.D. (2007) Toward a taxonomy of concept designs for improved robustness, Journal of Engineering Design, 18(2), 193-156.

Riquelme, N., von Lücken, C., \& Baran, B. (2015). Performance metrics in multi-objective optimization. In 2015 Latin American Computing Conference (CLEI) (pp. 1-11). IEEE.

Rötzer, S., Thoma, D., \& Zimmermann, M. (2020). Cost Optimization of Product Families Using Solution Spaces. In Proceedings of the Design Society: DESIGN Conference (Vol. 1, pp. 1087-1094). Cambridge University Press.

Sigurdarson, N., Eifler, T., \& Ebro, M. (2019). Functional Trade-offs in the Mechanical Design of Integrated Products - Impact on Robustness and Optimisability. Proceedings of the 20th International Conference on Engineering Design.

Suh, N.P. (2001) Axiomatic Design: Advances and Applications. United States, OUP.

Taguchi, G., Chowdhury, S. \& Wu, Y. (2005) Taguchi's Quality Engineering Handbook, Wiley \& Sons.

Yannou, B., Troussier, N., Chateauneuf, A., Boudaoud, N. \& Scaravetti, D. (2007) Design exploration, robust design and reliable design: Three successive and complementary approaches. International Conference on Engineering Design, ICED’07. 